\title{
TITLE:
}

\section{Cryogenic InSb detector for radiation measurements}

AUTHOR(S):

Kanno, Ikuo; Yoshihara, Fumiki; Nouchi, Ryo; Sugiura, Osamu; Nakamura, Tatsuya; Katagiri, Masaki

\section{CITATION:}

Kanno, Ikuo ...[et al]. Cryogenic InSb detector for radiation measurements. REVIEW OF SCIENTIFIC INSTRUMENTS 2002, 73(7): 2533-2536

\section{ISSUE DATE:}

2002-07

URL:

http://hdl.handle.net/2433/39806

\section{RIGHT:}

Copyright 2002 American Institute of Physics. This article may be downloaded for personal use only. Any other use requires prior permission of the author and the American Institute of Physics. 


\title{
Cryogenic InSb detector for radiation measurements
}

\author{
Ikuo Kanno, Fumiki Yoshihara, and Ryo Nouchi \\ Graduate School of Engineering, Kyoto University, Sakyo Kyoto 606-8501, Japan \\ Osamu Sugiura \\ Graduate School of Science and Engineering, Tokyo Institute of Technology, Meguro, Tokyo 152-8552, \\ Japan \\ Tatsuya Nakamura and Masaki Katagiri \\ Japan Atomic Energy Research Institute, Tokai, Ibaraki, 319-1195, Japan
}

(Received 26 October 2001; accepted for publication 21 March 2002)

\begin{abstract}
The energy spectra of ${ }^{241} \mathrm{Am}$ alpha particles were measured by a detector employing the compound semiconductor InSb at an operating temperature below $4.2 \mathrm{~K}$. The fabrication method and currentvoltage curves are shown. Though the energy resolution of the detector is not discussed in this article, this is the first report on an InSb radiation detector. (c) 2002 American Institute of Physics.

[DOI: $10.1063 / 1.1484238]$
\end{abstract}

\section{INTRODUCTION}

Radiation detectors, especially detectors for $\mathrm{x}$ rays and gamma rays with very high energy resolutions, are required in the research fields of pure physics such as x-ray astronomy and those of industrial applications. The requirement for energy resolution is some $\mathrm{eV}$ for $6 \mathrm{keV} \mathrm{x}$-rays, an energy resolution that cannot be achieved by conventional semiconductor detectors such as $\mathrm{Si}(\mathrm{Li})$ and $\mathrm{Ge}$ detectors, their theoretical limits of energy resolutions being nearly $120 \mathrm{eV}$ for $6 \mathrm{keV} \mathrm{x}$ rays.

To meet the requirement described above, detectors employing superconducting materials have been under extensive study. Theoretically, the energy resolution of superconducting radiation detectors should be only some $\mathrm{eV}$ for 6 $\mathrm{keV} \mathrm{x}$ rays. In practice, this energy resolution can be achieved only by superconducting radiation detectors with small active areas of which the typical dimension of the active area is $100 \mu \mathrm{m} \times 100 \mu \mathrm{m}$, an active area of less than $1 / 10000$ of that of conventional semiconductor detectors.

Inherently, superconducting radiation detectors cannot have large active areas. This is due to the high capacitance per unit area for superconductor-insulator-superconductor (SIS) tunnel junction detectors, and the heat capacitanceenergy resolution relationship for normal metal-insulatorsuperconductor (NIS) tunnel junction detectors and transition edge sensors (TESs). ${ }^{1}$ Another disadvantage of superconducting radiation detectors is their small absorber thickness. With a submicrometer superconducting layer in SIS detectors, and with some micrometers of a normal metal layer in NIS detectors and TESs, $x$ rays and gamma rays with energies higher than some tens of keVs cannot be absorbed efficiently.

However, for measurements of $6 \mathrm{keV} x$ rays in an astronomical application, superconducting radiation detectors with a small active area and thin absorbers can be excellent detectors. In industrial applications, which require detection of higher energy $x$ rays and gamma rays in short time periods, superconducting radiation detectors do not appear to be promising. We need, therefore, to develop other types of radiation detectors with larger active areas, and with energy resolutions higher than conventional semiconductor ones for advanced $\mathrm{x}$-ray applications in industry.

$\mathrm{McHarris}^{2}$ showed the possibility of the compound semiconductor $\mathrm{InSb}$ as a substrate for a radiation detector with high energy resolution. The band gap energy of $\mathrm{InSb}$ is 0.165 $\mathrm{eV}, 1 / 6$ of that of $\mathrm{Si}$, and $1 / 4$ of that of $\mathrm{Ge}$, while the mobility of its electrons is $78000 \mathrm{~cm}^{2} \mathrm{~V}^{-1} \mathrm{~s}^{-1}, 40$ and 20 times greater than those of $\mathrm{Si}$ and $\mathrm{Ge}$, respectively. The mobility of holes is $750 \mathrm{~cm}^{2} \mathrm{~V}^{-1} \mathrm{~s}^{-1}$, which while not very outstanding is 1.5 times greater than that of $\mathrm{Si}$. The high atomic numbers of In (49) and $\mathrm{Sb}$ (51), and its high density $\left(5.78 \mathrm{~g} \mathrm{~cm}^{-3}\right)$ make InSb very attractive for photon detection. Although $\mathrm{McHarris}$ pointed out these favorable features of InSb, and it has been employed in devices for Hall resistivity measurement, and for detectors of infrared, no work has been reported on using InSb for a radiation detector, except as an absorber in superconducting detectors. ${ }^{3,4}$

In this article, our first attempt to make an InSb radiation detector is described. The method of fabricating an InSb detector and the current-voltage curves of the detector are noted. Energy spectra of ${ }^{241} \mathrm{Am}$ alpha particles measured by this InSb detector are shown as functions of the operating temperature, the shaping time of the main amplifier, and the applied bias voltage. Our goal here is to show the use of an $\mathrm{InSb}$ detector to measure $\mathrm{x}$ rays with a high energy resolution. However, this is just the first report on radiation measurement by a detector made of InSb, and for ease of use in this we employed alpha particles for radiation measurements to judge if the detector actually works or not. The energy resolution of the InSb detector is not discussed at this point, this being just our first attempt at such a fabrication.

\section{EXPERIMENT}

\section{A. Device fabrication}

We fabricated a surface barrier-type detector, with a rectifying Schottky contact on the front surface of an InSb sub- 


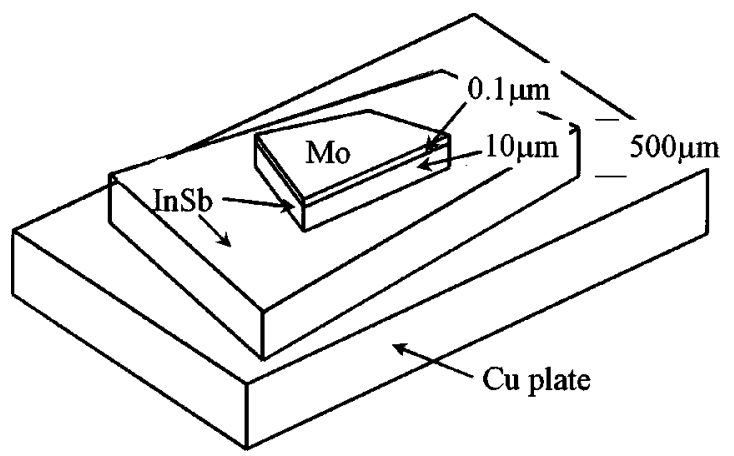

FIG. 1. Schematic drawing of the InSb detector.

strate, and an Ohmic contact on the back side. The InSb employed was a $p$-type wafer (Wafer Technology Ltd., England) a diameter of 2 in., thickness of $500 \mu \mathrm{m}$, and with a Ge dopant concentration of $3.5 \times 10^{15} \mathrm{~cm}^{-3}$. The resistivity of the InSb substrate at $77 \mathrm{~K}$ was $0.29 \Omega \mathrm{cm}$.

The InSb substrate (nearly $4 \mathrm{~mm} \times 6 \mathrm{~mm}$ ) was etched using a mixture of nitric and lactic acids (1:10) for $5 \mathrm{~min}$. On this etched surface, a Schottky electrode was made by evaporating Mo with a thickness of $0.1 \mu \mathrm{m}$. Next, a mesa electrode was defined using a photoresist mask, and formed by etching Mo and InSb with suitable etchants with mixtures of nitric and phosphoric acids and of nitric and lactic acids for Mo and $\mathrm{InSb}$, respectively. The dimensions of the mesa were nearly $2 \mathrm{~mm} \times 3 \mathrm{~mm}$, with a thickness of $10 \mu \mathrm{m}$. Finally, the processed wafer was mounted by $\mathrm{In}$ solder on a $\mathrm{Cu}$ plate having an Ohmic contact on the back surface. A schematic drawing of the detector is shown in Fig. 1.

\section{B. Measurement of current-voltage curves}

Current-voltage $(I-V)$ curves were measured by the usual method of connecting the InSb detector, a voltage supply (Yokogawa 7651), and a digital multimeter (Keithley $197 \mathrm{~A})$ in series. The $I-V$ curves were measured at several temperatures from 4.2 to $77 \mathrm{~K}$. Measured $I-V$ curves, double Schottky-like, are shown in Fig. 2, where a positive $x$ value corresponds to the positive applied voltage on the $\mathrm{Cu}$

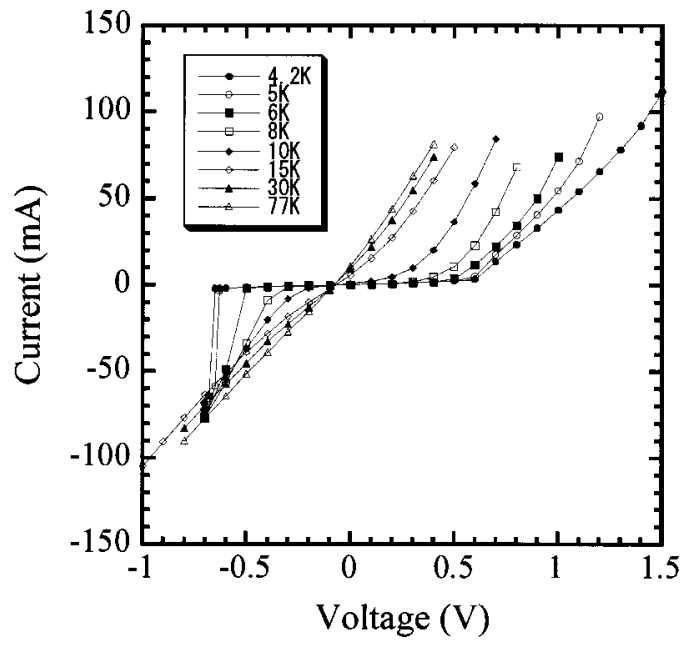

FIG. 2. Current-voltage curves of the InSb detector. The operating temperatures are shown in the figure.

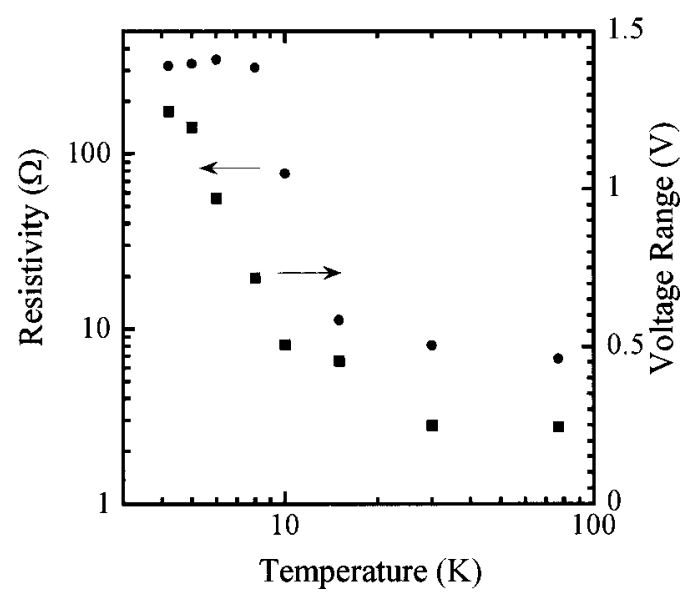

FIG. 3. Resistivity (solid circles) and voltage range (solid squares) of the center regions of the $I-V$ curves of Fig. 2.

electrode. The resistivity at the voltage around $0 \mathrm{~V}$, and the voltage range for which the same resistivity holds, were estimated for each $I-V$ curve. The summarized results are shown in Fig. 3.

\section{Alpha particle measurement}

The InSb detector was mounted on the $0.3 \mathrm{~K}$ stage of a refrigerator (Infrared Co.) and an electrodeposited ${ }^{241} \mathrm{Am}$ alpha particle source placed some millimeters from the surface of the detector. The alpha particles (mainly $5.4 \mathrm{MeV}$ in energy) were collimated at nearly $4 \mathrm{~mm}$ diam.

The electronic circuit was a conventional one as shown in Fig. 4. The preamplifier used was a Canberra 2004 with the originally connected $100 \mathrm{M} \Omega$ resistor replaced by a 2 $\mathrm{M} \Omega$ resistor.

In this electronic circuit, the voltage applied to the detector $V_{d}$ was calculated by the following equation:
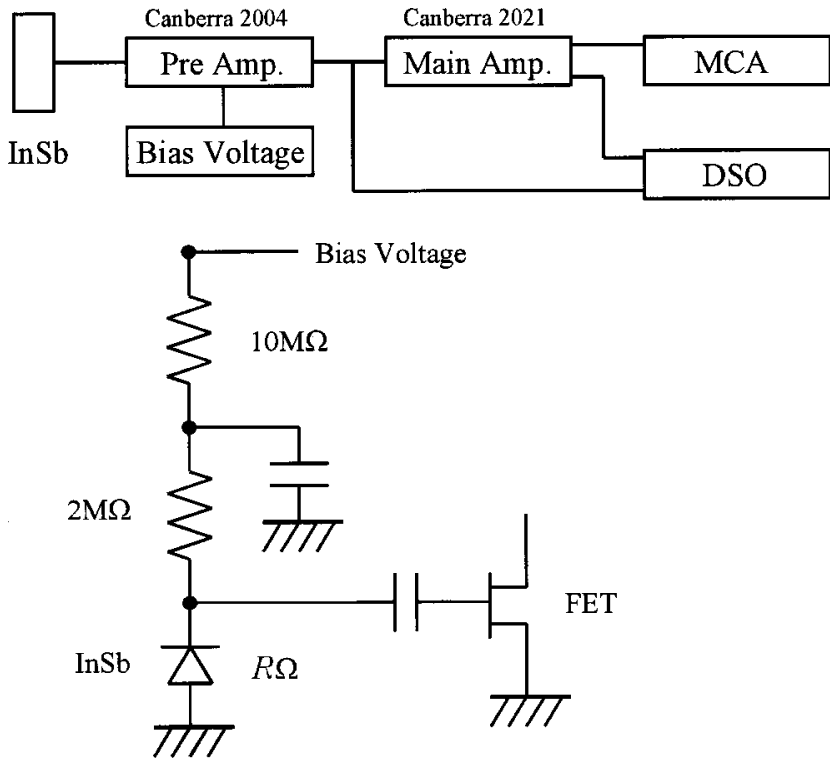

FIG. 4. Electronic circuit for alpha particle measurement (MCA: multichannel analyzer, DSO: digital storage oscilloscope). Details of the input part of the preamplifier are shown. 

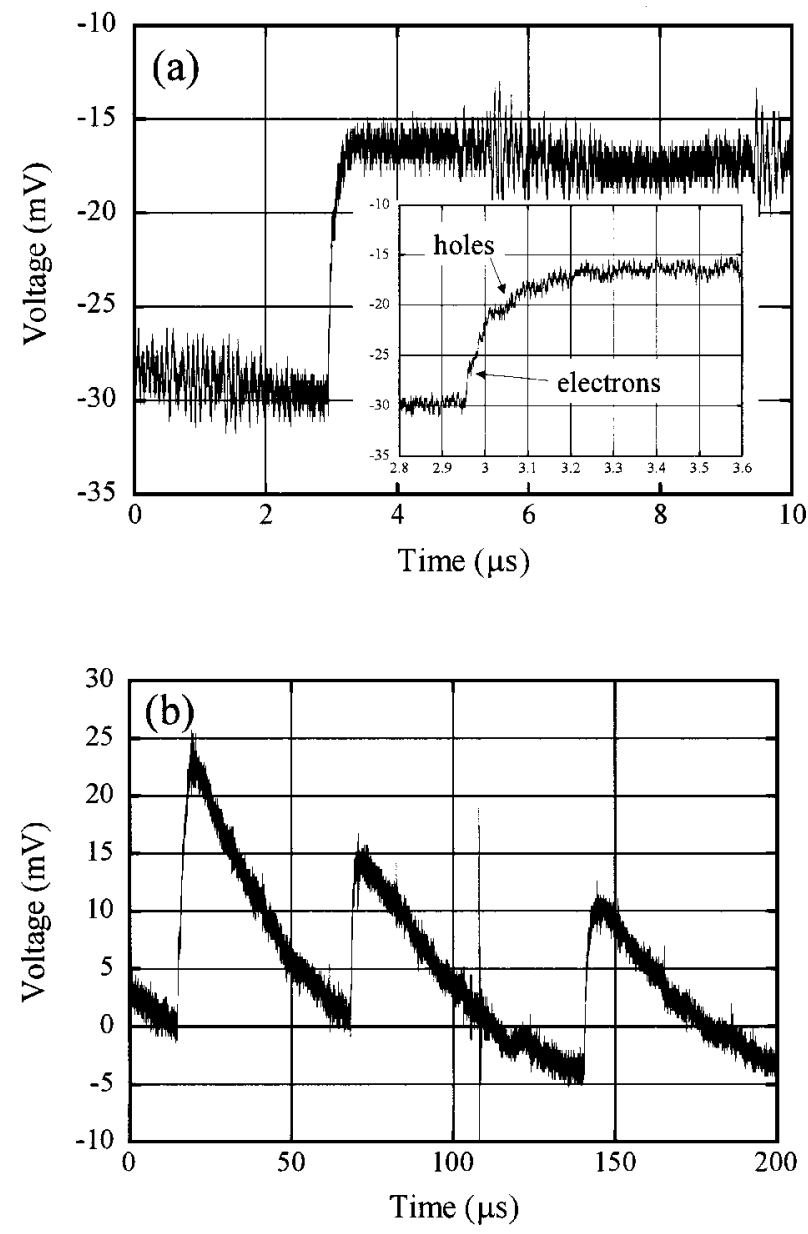

FIG. 5. Preamplifier output pulses at temperatures (a) $0.5 \mathrm{~K}$ and (b) $4.2 \mathrm{~K}$.

$$
V_{d}=V_{i}+\frac{R \Omega}{(10+2) M \Omega+R \Omega} \cdot V_{b},
$$

where, $V_{i}$ is the inherent voltage, $V_{b}$ is the output voltage of the bias voltage, and $R$ is the resistivity of the InSb detector at the operating temperature. When a bias voltage of $100 \mathrm{~V}$ was applied to the InSb detector at an operating temperature of $4.2 \mathrm{~K}$, Eq. (1) gives a very small voltage, such as $V_{i}$ $+2.5 \mathrm{mV}$, substituting $R$ with $300 \Omega$ as shown in Fig. 3. The determination of $V_{i}$ was difficult, however, it was estimated as less than $1 \mathrm{~V}$, following a number of examples of semiconductor devices.

The preamplifier and main amplifier output pulses of InSb were observed by digital storage oscilloscope, and the pulse height spectra measured by multichannel analyzer (MCA).

The output pulses of the preamplifier at the operating temperatures of (a) $0.5 \mathrm{~K}$ and (b) $4.2 \mathrm{~K}$ are shown in Fig. 5. At $0.5 \mathrm{~K}$ the rise time of the pulse was nearly $250 \mathrm{~ns}$, but with a very long decay time. In the inset of Fig. 5(a), fast and slow components, which we think correspond to the contributions of electrons and holes, respectively, can be observed in the rising part of the pulse. At $4.2 \mathrm{~K}$, the rise time was nearly $1 \mu \mathrm{s}$, and the pulse decay shorter than that at $0.5 \mathrm{~K}$. As the operating temperature rose from 0.5 to $4.2 \mathrm{~K}$, the slow component of the output pulse became dominant.
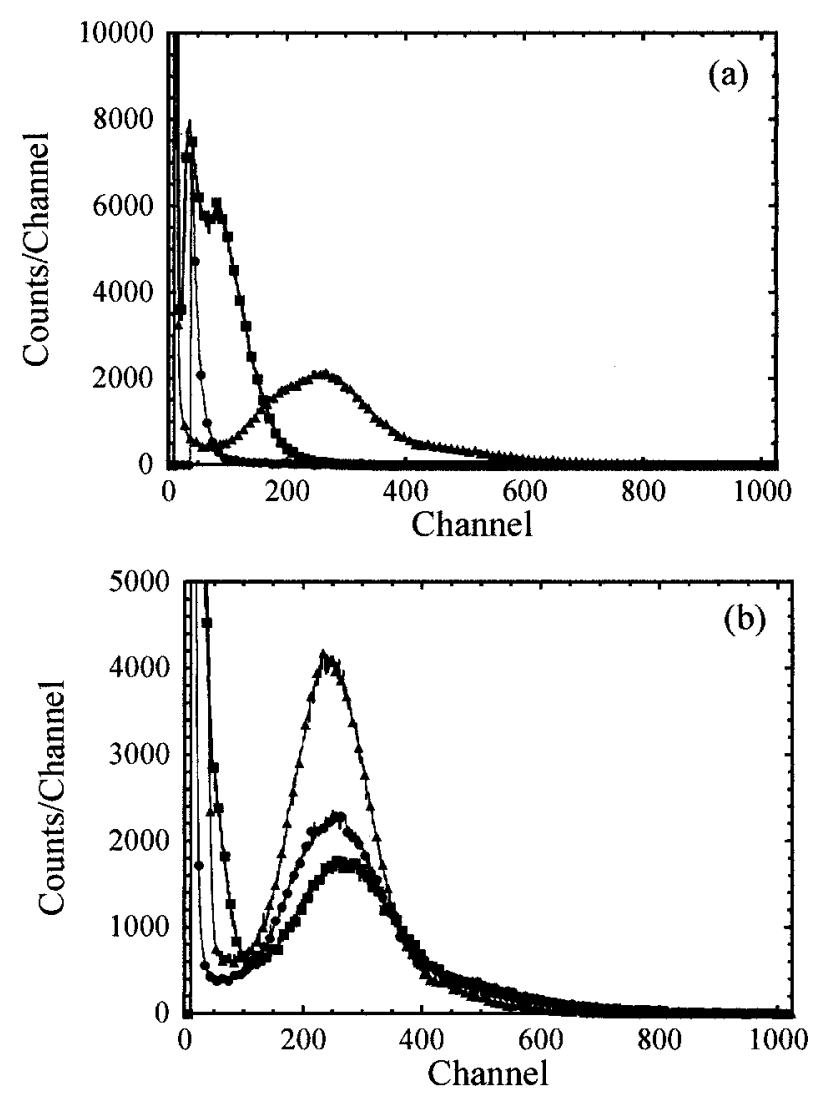

FIG. 6. Energy spectra of ${ }^{241} \mathrm{Am}$ alpha particles measured at operating temperature, shaping time, and applied bias voltage $V_{b}$ of (a) $0.5 \mathrm{~K}, 0.25 \mu \mathrm{s}, 1$ $\mathrm{V}$ (solid circles), $3.5 \mathrm{~K}, 0.25 \mu \mathrm{s}, 0 \mathrm{~V}$ (solid squares), and $3.5 \mathrm{~K}, 4 \mu \mathrm{s}, 0 \mathrm{~V}$ (solid triangles); (b) $4.2 \mathrm{~K}, 4 \mu \mathrm{s}, 0.5 \mathrm{~V}$ (solid circles), $4.2 \mathrm{~K}, 4 \mu \mathrm{s}, 50 \mathrm{~V}$ (solid squares), and $4.2 \mathrm{~K}, 1 \mu \mathrm{s}, 50 \mathrm{~V}$ (solid triangles), respectively. The voltage actually applied to the detector $V_{d}$ is calculated by Eq. (1).

We measured, $5 \mathrm{~min}$ for each measurement, the energy spectra of alpha particles with the rising of the operating temperature. The measured pulse height spectra are shown in Figs. 6(a) and 6(b) and the applied bias voltage and shaping time of the main amplifier are in the legend.

\section{DISCUSSION}

\section{A. Current-voltage curve of the InSb detector}

Double-Schottky-like $I-V$ curves were obtained at temperatures below $10 \mathrm{~K}$, Fig. 2 . We think this was the result of an incomplete Ohmic electrode showing Schottky-like characteristics at these lower temperatures.

Estimations of resistivity and voltage range became difficult at temperatures above $15 \mathrm{~K}$, Fig. 3, and could have had large errors. However, we note the drastic change of resistivity between 10 and $15 \mathrm{~K}$. That the resistivity showed a saturated behavior below $8 \mathrm{~K}$ is encouraging for the practical application of InSb detectors. In the future, with a better Schottky electrode, resistivity and voltage range at low temperatures would increase and a thicker depletion layer could be achieved.

\section{B. Energy deposition of alpha particles}

According to the semi-empirical formula of Ziegler et al. related to the range and energy loss of ions, ${ }^{5}$ the energy 
of alpha particles with an initial energy of $5.4 \mathrm{MeV}$ after passing the Mo layer was $5.36 \mathrm{MeV}$. The range of the alpha particles in the InSb substrate was calculated as $0.19 \mu \mathrm{m}$. On the other hand, the thickness of the depletion layer of the $\mathrm{InSb}$ detector was estimated as $0.14 \mu \mathrm{m}$, using a resistivity of the InSb substrate of $0.29 \Omega \mathrm{cm}$ at $77 \mathrm{~K}$ the hole mobility as $750 \mathrm{~cm}^{2} \mathrm{~V}^{-1} \mathrm{~s}^{-1}$, and assuming the inherent voltage as 0.5 V. ${ }^{6}$ We could not tell if the depletion layer thickness was greater than the range of alpha particles at a lower temperature, at which resistivity of the substrate could be greater than that at $77 \mathrm{~K}$.

In practical use for $\mathrm{x}$-ray measurements, a thick depletion layer is necessary for efficient detection. The fabrication of an InSb substrate with high resistivity is a key technology in detector development.

\section{Charge collection process in the InSb detector}

On the digital storage oscilloscope, we observed a strong electronic noise in the preamplifier output when a bias voltage was applied to the InSb detector at $0.5 \mathrm{~K}$. This phenomenon was taken as being the electron avalanche due to energetic electrons under a stronger electric field with a long mean free path and high mobility at low temperature. To avoid this phenomenon, an $n$-type substrate should be employed; in an $n$-type semiconductor detector with a rectifying electrode, electrons experience a weaker electric field as they approach the positive electrode. The velocity they would obtain has limitations, which is not enough to induce an electronic avalanche. On the other hand, holes will have a stronger electric field as they move to the negative electrode. However, the hole velocity will not be too high due to their mobility, less than $1 / 100$ of that of an electron.

The difference of preamplifier outputs at 0.5 and at 4.2 $\mathrm{K}$, as shown in Figs. 5(a) and 5(b), was due to the movement of electrons and holes. At $0.5 \mathrm{~K}$, electrons quickly arrived at the positive electrode due to their greater mobility at low temperatures, and holes were captured and released very slowly. At higher temperatures, the movement of electrons became slower, while the release of holes occurred soon after they were captured.

The pulse height spectrum measured at $0.5 \mathrm{~K}$ was in the smaller channels of MCA as shown in Fig. 6(a). At a low temperature such as $0.5 \mathrm{~K}$, most of the electrons and holes created by the energy deposition of the alpha particles froze out. This is closely related to the output pulse of the preamplifier. At a temperature of $3.5 \mathrm{~K}$, a slow component of the carriers appeared and had a higher pulse height with a shaping time of $4 \mu \mathrm{s}$. The pulse height with an applied voltage of $50 \mathrm{~V}$ was slightly higher than that with $0.5 \mathrm{~V}$, Fig. 6(b). According to Eq. (1) the difference of $V_{d}$ between these two bias voltages was nearly $1.2 \mathrm{mV}$. This indicated that the electrons and holes created by the alpha particles were under the influence of an electric field, i.e., the InSb detector behaved as a semiconductor detector. While the shaping time of $1 \mu \mathrm{s}$ may be a better condition for measurement, we still have to fabricate InSb detectors with better rectifying characteristics to study their operating conditions, and their charge collection process.

\section{ACKNOWLEDGMENTS}

Part of this work was performed with the support of the Venture Business Laboratory Project, Kyoto University.

${ }^{1}$ M. Nahum, M. Martinis, and S. Castles, J. Low Temp. Phys. 93, 733 (1993).

${ }^{2}$ Wm. C. McHarris, Nucl. Instrum. Methods Phys. Res. A 242, 373 (1986).

${ }^{3}$ D. J. Gorldie, A. M. Swift, N. E. Booth, and G. L. Salmon, Nucl. Instrum. Methods Phys. Res. A 344, 592 (1994).

${ }^{4}$ R. J. Gaitskell, L. C. Angrave, N. E. Booth, A. D. Hahn, G. L. Salmon, and A. M. Swift, Nucl. Instrum. Methods Phys. Res. A 370, 250 (1996).

${ }^{5}$ J. F. Ziegler, J. P. Biersack, and U. Littmark, The Stopping and Range of Ions in Matter (Pergamon, New York, 1985), Vol. 1.

${ }^{6}$ G. F. Knoll, Radiation Detection and Measurements (Wiley, New York, 2000). 